\title{
Simulation of Motion of Micrometer Sized Droplets of Dielectric Water-based Paint under Air Flow and Electric Field
}

\author{
Wei-Wei Xu and Yong-Ming Zhang
}

\begin{abstract}
In the present study, the motion of the micrometer sized droplets of dielectric water-based paint in the air is investigated numerically. The effects of gravity, the inlet velocity of air flow and applied external positive DC electric field have been taken into account. In the first part, the motion of the droplets is simulated. It is found that the effect of gravity and inlet velocity of air flow can be neglected under our conditions. In the second part, the motions of the droplets under the external electric field are simulated to investigate the effect of electric field. Our results show that the external positive electric field can result the fall speed increase comparison with the condition under no electric field. It is also observed that impact time decreases as electric potential increases. The simulation results show that the Navier-Stokes equations coupled with other fields provide an effective approach to analyze the dynamic problems for liquid droplet.
\end{abstract}

Index Terms-Droplet, level set method, electric field, water-based paint.

\section{INTRODUCTION}

Due to the wide applications in micro fluidics devices [1]-[3], mist [4], [5], and spray [6], a great deal of studies on multiphase flows have been done and attracted much attention in the past several decades. After the pioneering work of Worthington [7], more attention on droplet research is around the droplet dynamic behaviours by experimental measurements and numerical simulations in recent years.

In the aspect of experimental study, Reference [8] observed that the maximal spreading diameter of elastic fluid droplets impact onto rough surfaces could be as much as twice larger than those on smooth surfaces. Reference [9] demonstrated an empirical equation for a milimeter sized droplet about the fall speed and fall height by using a shadowgraph method with a rapid CCD camera system. Reference [10] reported that there was a linear relation between the contact line velocity and the relationship between fall speed and fall height by using a high-speed video camera. Reference [11] demonstrated that the droplet expansion process can be controlled through variation of the ion injection current and /or discharge exposure time. Reference [12] performed an experiment for dielectric droplets deposited on a superhydrophobic surface

Manuscript received November 4, 2016; revised January 5, 2017. This work was supported in part by the Fundamental Research Funds for the Central Universities under Grant WK2320000032 and the Joint Funds of the National Natural Science Foundation of China under Grant U1233102.

The authors are with the State Key Laboratory of Fire Science, University of Science and Technology of China, Jinzhai Road No. 96, 230026, Hefei, China (e-mail: xuweiwei2005@163.com, zhangym@ustc.edu.cn). and under a uniform electric field. Their result showed that the change in the droplet shape is described by a linear dependence of its eccentricity under the electric field.

On the other side, various numerical investigations have also been introduced to the droplet dynamic behaviours research. Reference [13] reported that a higher Weber number could lead to a longer contact length for a single droplet by using LBM method. Reference [14] assessed the dielectric droplets dynamic behaviour by simulation analysis based on the Navier-Stokes equation, the Poisson equation and the Nernst-Planck equations. Their results showed that the predictions are reliable even at considerably large deformations.

Although a lot of work has been carried out for investigation of droplet dynamic behaviours, some problems are still not resolved. For instance, micrometer or even smaller sized droplets motion with initial velocity within the air flow have been limited. Moreover, coupled with electric field and gravity for the droplet motion in the air flow has not been considered. Therefore, the more interest is shown to study the fundamental understanding of effects of gravity, air flow velocity, electric field and electric potential to the motion of the micrometer sized droplets of dielectric water-based paint in the air. In this paper, we present a numerical simulation experimental study of the motion effect of micrometer sized droplets of dielectric water-based paint through the air.

\section{Procedure FOR PAPER SUbMission Problem DESCRIPTION AND BOUNDARY CONDITIONS}

Fig. 1 illustrates a schematic two dimension (2D) axisymmetric model for a micrometer sized droplet of dielectric water-based paint to be analysed in the following research. The initial radius $\left(r_{0}\right)$ of droplet is $20 \mathrm{um}$. A droplet of dielectric water-based paint is placed in a flow field, under. In the numerical study, the droplet is kept at the center line of the bottom of steel surface with a 450 um distance vertical to the bottom steel surface. After applying different electric potentials to the parallel steel surfaces, a positive DC uniform electric field is generated along the vertical direction. For the sake of simplicity, the following assumptions are made in the following research:

(a) The two phase fluids are incompressible

(b) The effect of magnetic induction is neglected

(c) There is no charge except the interface separating the two phase fluids

(d) There is no mass loss 
(e) The shape of the droplet is spherical at the initial stage

TABLE I: PARAMETERS AND MATERIAL IN THE SIMULATION

\begin{tabular}{ll}
\hline \hline Droplet material & Water-based paint \\
Gas phase & Air \\
Density of the droplet $\left(\rho_{\text {droplet }}\right)$ & $1320\left[\mathrm{~kg} / \mathrm{m}^{3}\right]$ \\
Density of air $\left(\rho_{\text {air }}\right)$ & $1.225\left[\mathrm{~kg} / \mathrm{m}^{3}\right]$ \\
Interfacial tension coefficient of & $0.0648[\mathrm{~N} / \mathrm{m}]$ \\
droplet & $0.179[\mathrm{~Pa} \cdot \mathrm{s}]$ \\
Viscosity of droplet $\left(\mu_{\text {droplet }}\right)$ & $1.7894 \times 10^{-5}[\mathrm{~Pa} \cdot \mathrm{s}]$ \\
Viscosity of air $\left(\mu_{\text {air }}\right)$ & $20[\mu \mathrm{m}]$ \\
Radius of droplet $\left(r_{0}\right)$ & $9.8\left[\mathrm{~m} \cdot \mathrm{s}^{-2}\right]$ \\
Gravitational acceleration $(g)$ &
\end{tabular}

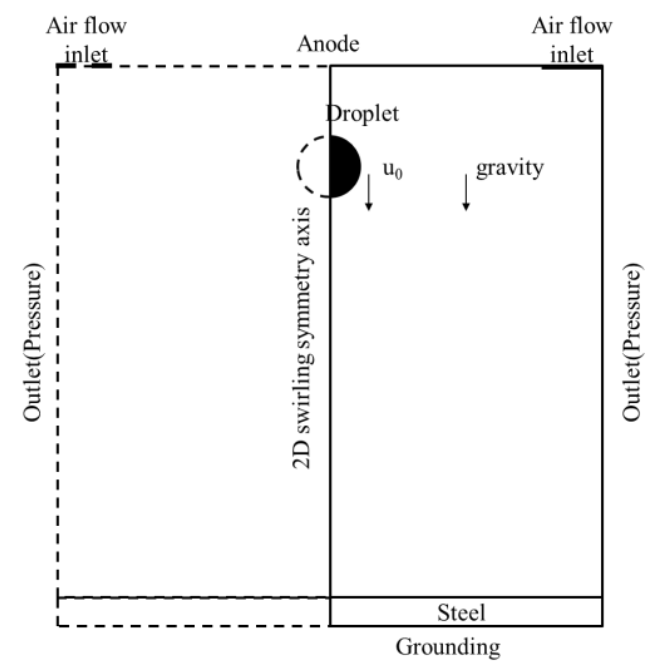

Fig. 1. Schematic diagram of the simulation domain and boundary. The droplet moves along the vertical direction.

\section{MATHEMATICAL FOURMULATIONS}

\section{A. Governing Equations for Two-Phase Flow}

In the incompressible two phase flow system, the motion of fluid is governed by equations of Navier-Stokes, which is validated in [15], [16]. To take into consideration of the surface tension, gravity and the electric stress, the Navier-Stokes can be expressed as:

$\rho \frac{\partial u}{\partial t}+\rho(u \nabla)=\nabla\left[-p I+\mu\left(\nabla u+(\nabla u)^{T}\right)\right]+F_{s t}+\rho g+F_{e s}$

$\nabla \quad u=0$

where $u$ denotes fluid velocity, $\rho$ is the fluid density, $g$ is the gravitational acceleration, $\mu$ is the dynamic viscosity, $F_{e s}$ is the electric stress, $F_{s t}$ is the surface tension, I is identity matrix and $p$ is the pressure. Without electric potentials, $F_{e s}$ will be neglected.

\section{B. Conservative Level Set Method}

The interface in a level set method is captured by a level set function $\phi$. The function $\phi$ is governed by:

$$
\frac{\partial \phi}{\partial \mathrm{t}}+\nabla \cdot(\phi u)=\alpha \nabla \cdot\left(\varepsilon_{1 s} \nabla \phi-\phi(1-\phi) \frac{\nabla \phi}{|\nabla \phi|}\right) .
$$

where $\varepsilon_{1 s}$ is the parameter controlling the interface thickness and $\alpha$ is the reinitialized parameter. In general, inside the droplet $\phi=1$ and ambient fluid $\phi=0$. The interface is represented by the 0.5 contour of the level set function $(\phi=0.5)$.

The surface tension force is computed as:

$$
F_{s t}=\nabla T=\nabla \cdot\left[\left(\sigma\left(I-n n^{T}\right)\right) \delta\right] .
$$

where $I$ is the identity matrix, $n$ is the interface normal, and $\sigma$ is the surface tension, and $\delta$ is the Dirac delta function that is zero except at the interface separating the two phase fluids. The interface normal is calculated as:

$$
n=\frac{\nabla \phi}{|\nabla \phi|} \text {. }
$$

The delta function $\delta$ is approximated by a smooth function defined by:

$$
\delta=6|\phi(1-\phi)||\nabla \phi|
$$

\section{Governing Equations for Electric Field}

The droplets will deform due to the electric forces. In the case of absence of any time-varying magnetic field, the curl of the electric field is zero $(\nabla \times E=0)$ and the electric field can be descripted by the electric potential $U$ :

$$
E=-\nabla U \text {. }
$$

In this two phase fluid system, we assume that the electric relaxation time is shorter than the time scale of the fluid motion, and the electric conductivity is constant for water-based paint and air. The governing equation for the potential can be expressed in each medium as follows:

$$
\begin{aligned}
& \nabla^{2} U=0 \\
& D=\varepsilon_{0} \varepsilon_{r} E .
\end{aligned}
$$

where $D$ is the electric displacement, $\varepsilon_{0}$ is the permittivity of vacuum, $\varepsilon_{r}$ is the relative permittivity. Based on the former assumption, the electric stress can be calculated by taking the divergence of the Maxwell stress tensor, which couples electrostatic and hydrodynamic phenomena. The Maxwell stress tensor can be defined as:

$$
T M_{i j}=\varepsilon_{0} \varepsilon_{r} E_{i} E_{j}-\frac{1}{2}\left(\varepsilon_{0} \varepsilon_{r} E^{2}\right) \delta_{i j} .
$$

The momentum equation is modified by inserting the electric force $F_{e s}$, which can be determined by calculating the divergence of the Maxwell stress tensor TM:

$$
F_{e s}=\nabla T M \text {. }
$$

The relative permittivity for water-based paint and air is constant, but different. In order to have all the physical properties at the interface, the relative permittivity $\left(\varepsilon_{r}\right)$ can be defined by the volume fractions of each fluid, $V f 1$ and $V f 2$ :

$$
\varepsilon_{r}=\varepsilon_{r 1} \cdot V f 1+\varepsilon_{r 2} \cdot V f 2 .
$$

where $\varepsilon_{r 1}$ and $\varepsilon_{r 2}$ denote the relative permittivity of air and water-based paint. Inside the droplet, $V f 1=0$ and $V f 2=1$, whereas outside the droplet, $V f l=1$ and $V f 2=0$.

\section{DETERMINATION OF DROPLET VELOCITY}

For each simulation setting, the droplet velocity is determined as displayed in Fig. 2. The droplet position is obtained by matlab image analysis. As the delay between the 
two frames $\Delta t\left(\Delta t=t_{i}-t_{i-1}\right)$ is known, the spatial difference $\Delta y i$ readily provides the droplet velocity:

$$
u_{t i}=\frac{\Delta y_{i}}{\Delta t} .
$$

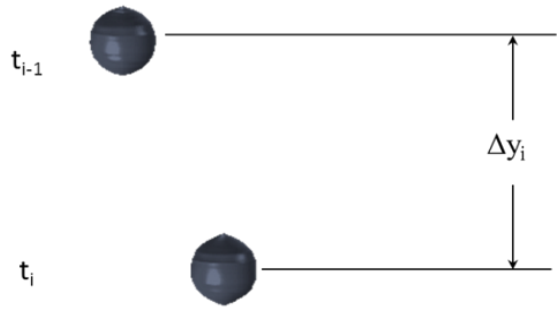

Fig. 2. Determination of velocity of droplet motion in the air. All images shown are for equal droplet settings. Two frames are used to determine the droplet translation $\Delta y$ i. The translation is used to obtain the droplet velocity. To reduce the uncertainty, the time scale between two adjacent images is $0.01 \mathrm{~ms}$.

\section{RESULTS AND DISCUSSION}

In this section, the motion of the micrometer sized droplets of dielectric water-based paint under air flow and electric field is discussed in detail.

\section{A. Effect of Gravity}

In order to investigate the effect of gravity on the motion for the droplets, the inlet velocity of air flow $\left(u_{\text {inlet_air }}\right)$ is 0 , as well as the electric potential. Fig. 3 shows the relationship between fall height and fall speed under our simulation conditions. From Fig. 3, we can see that the gravity can be neglected for our simulated sized droplet.

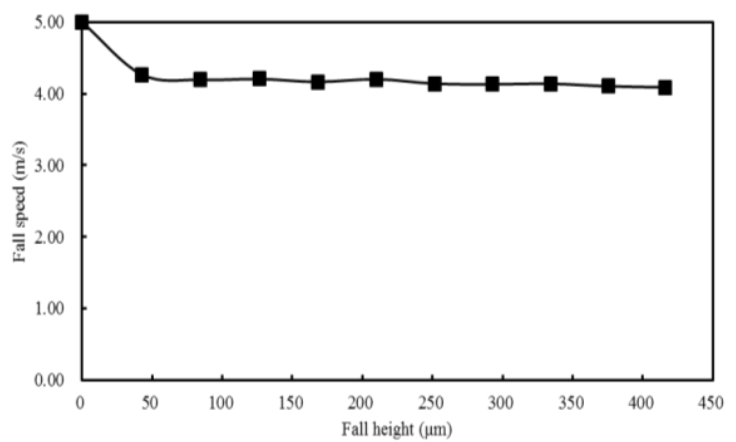

Fig. 3. Comparison of experimental data of fall heights and fall speeds with gravity and without gravity. (-) gravity is taken into account; (匹) neglect the effect of gravity.

Simply integrated the equation of droplet motion for a fixed friction coefficient $\left(c_{f}\right)$ under higher Reynolds numbers in our simulation, we can obtain an empirical equation:

$$
\begin{gathered}
u_{i}=\sqrt{17.2+7.79 e^{-\alpha H}} . \\
\alpha=\frac{3}{8} \frac{c_{f} \rho_{\text {air }}}{\rho_{\text {droplet }} \cdot r_{0}} .
\end{gathered}
$$

where $H(\mu \mathrm{m})$ is the fall height of the droplets motion in the air. $c_{f}$ in the above equation has been adjusted to 0.0025 by the best fit to velocity obtained from numerical experiment at different fall heights.

\section{B. Effect of Inlet Velocity of Air Flow}

Fig. 4 shows, for different inlet velocity of air flow and gravity taken into consideration, the evolution of the fall velocity $u_{i}$ versus different fall heights. Fig. 4 shows that the fall speed data for the case of $u_{\text {inlet-air }}=10 \mathrm{~m} / \mathrm{s}$ coincide in the line for the case of $u_{\text {inlet-air }}=0 \mathrm{~m} / \mathrm{s}$, although there is a slight deviation at the last height. Therefore, the effect of inlet velocity of air flow for our simulation conditions can be neglected and the relationship between fall speed and fall height can be descripted as Eq. 12.

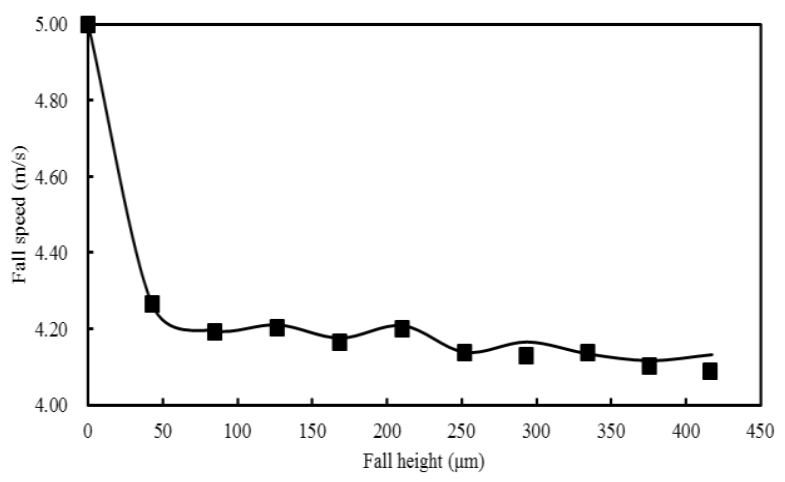

Fig. 4. Comparison of fall speed versus fall height under different inlet velocity of air flow. $(-) u_{\text {inlet-air }}=10 \mathrm{~m} / \mathrm{s},(\square) u_{\text {inlet-air }}=0 \mathrm{~m} / \mathrm{s}$.

\section{Effect of Electric Field}

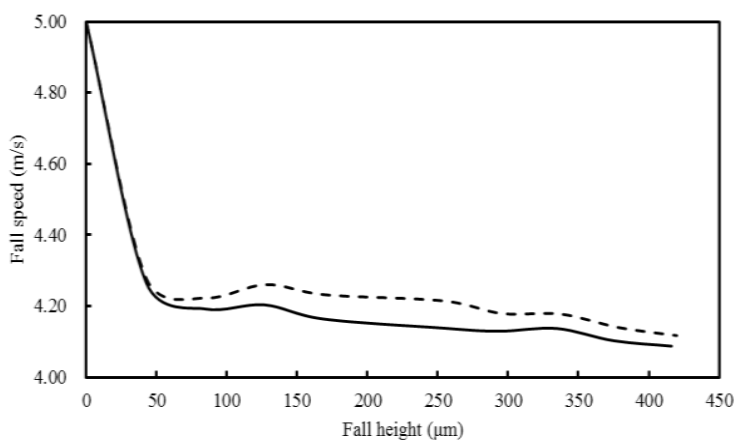

Fig. 5. Comparison of fall speed versus fall height with/without electric field (-) without electric field, (- - ) applied potential $U=1000 \mathrm{~V}$.

In this model, the droplets of dielectric water-based paint having radius $r_{0}$ move in the air with initial velocity $\mathrm{u}_{0}$, while the upper steel surface is given a potential $U=1000 \mathrm{~V}$ and time interval between the two adjacent pictures is $0.01 \mathrm{~ms}$. The droplet motion simulations are performed with the same static contact angle at initial time. In the presence of the electric forces, the droplets move in the air, and subsequently change shapes. In Fig. 5, the fall speed of the droplets at different fall heights under external electric field is compared with the droplet fall speed without electric field effect.

As shown in Fig. 5, the numerically predicted droplet fall speed versus fall height with and without external electric field. We can see that the electric field has an important effect for the droplets fall speed in the air. Fig. 5 shows that the external positive electric field can result the fall speed increase comparison with the condition under no electric field.

\section{Effect of Electric Potential}

In this model, the droplet of dielectric water-based paint is placed in the electric field with the same initial velocity $\mathrm{u}_{0}$ and radius $r_{0}$. The droplet motion simulations are carried out with series different electric potential. In order to reduce the 
uncertainty, the exact instant of motion has been settled on the time interval of $\Delta \mathrm{t}=0.002 \mathrm{~ms}$. The static contact angle is $30^{\circ}$.

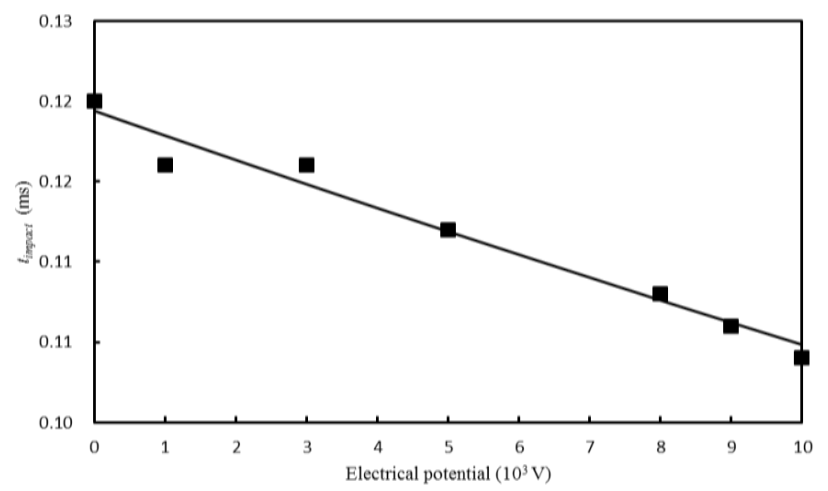

Fig. 6. Impact time versus applied electric potential. Square(a) is the numerical test value, solid line (-) is the best fit line corresponds to the best fit (Eq. 14).

In Fig. 6, the impacting time of the motion droplet is as a function of the applied electric potential. The solid line in Fig. 6 is the best fit of the numerical experimental data according to the exponential equation:

$$
t_{\text {impact }}=A \cdot \exp (B \cdot U) .
$$

where $A$ and $B$ is the fit constant, with $A=0.1194, B=-0.013$, $U$ is the applied electric potential. From Fig. 6, we can see the impact time $t_{\text {impact }}$ for numerical tested droplets decreases as the applied electric potential increases, which means increasing electric potential can increase the droplet motion speed.

\section{CONCLUSION}

Based on the Navier-Stokes equation, a series numerical investigation of the vertical motion of the micrometer sized droplets of dielectric water-based paint between two horizontal steel surface under different conditions have been carried out. The new findings of this study, within different conditions investigated, are summarized as follows:

1) The gravity can be neglected for the motion of a $40 \mu \mathrm{m}$ dielectric water-based paint droplet or even smaller sized droplets motion in the air.

2) The effect of inlet velocity of air flow can be neglected.

3) Electric field has an important effect to the droplets fall speed.

4) Increasing the applied electric potential can decrease the impact time for droplets motion.

The results may have practical application for microfluidic research and control that rely on the droplets manipulation.

\section{ACKNOWLEDGMENT}

We acknowledge the help and technical support of Professor Jun Qin.

\section{REFERENCES}

[1] P. Cheng and H. Y. Wu, "Mesocale and microscale phase-change heat transfer," Advance Heat Transfer, vol. 39, pp. 461-563, Dec. 2006

[2] $\mathrm{H}$. Wu and P. Cheng, "Boiling instability in parallel silicon microchannels at different heat flux," Int. J. Heat Mass Transfer, vol. 47, pp. 3631-3641, Aug. 2004
[3] X. Quan, P. Cheng, and H. Wu, "Transition from annular flow to plug/slug flow in condensation of steam in microchannels," Int. J. Heat Mass Transfer, vol. 51, pp. 707-716, Feb. 2008

[4] T. Wang, J. L. Gaddis, X. Li, "Mist/steam heat transfer of multiple rows of impinging jets," Int. J. Heat Mass Transfer, vol. 48, pp. 5179-5191, Dec. 2005

[5] N. Kumari, V. Bahadur, M. Hodes, T. Salamon, P. Kolodner, A. Lyons, and S. V. Garimella, "Analysis of evaporating mist flow for enhanced convective heat transfer," Int. J. Heat Mass Transfer, vol. 53, pp. 3346-3356, Jul. 2010

[6] S. Freund, A. G. Pautsch, T. A. Shedd, and S. Kabelac, "Local heat transfer coefficients in spray cooling systems measured with temperature oscillation IR thermography," Int. J. Heat Mass Transfer, vol. 50 , pp. 1953-1962, May 2007

[7] A. M. Worthington, "On the forms assumed by drops of liquids falling vertically on a horizontal plate," in Proc. R. Soc. Lond., vol. 25, pp. 261-272, Jan. 1876

[8] L-H. Luu and Y. Forterre, "Giant drag reduction in complex fluid drops on rough hydrophobic surfaces," Phys. Rev. Lett., vol. 110, pp. 614-618, May 2013.

[9] R. Kai and F. Feuillebois, "Influence of Surface Roughness on Liquid Drop Impact,” J. Colloid Interf. Sci., vol. 203, pp. 16-30, Jul. 1998

[10] D. C. D. Roux and J. J. Cooper-White, "Dynamics of water spreading on a glass surface," J. Colloid Interf. Sci., vol. 277, pp. 424-436, Sep. 2004

[11] S. R. Mahmoudi, K. Adamiak, and G.S. P. Castle, "Spreading of a dielectric droplet through an interfacial electric pressure," in Proc. $R$. Soc. A, vol. 467, pp. 3 257-3271, Nov. 2011

[12] E. Bormashenko, R. Pogreb, T. Stein, G. Whyman, and M. Hakham-Itzhaq, "Electrostatically driven droplets deposited on superhydrophobic surfaces," Appl. Phys. Lett., vol. 95, pp. 264102, Dec. 2009

[13] J. Wu, J. J. Huang, and W. W. Yan, "Lattice Boltzmann investigation of droplets impact behaviors onto a solid substrate," Colloid. Surf. A: Physicochem. Eng. Aspects, vol. 484, pp. 318-328, Nov. 2015

[14] G. Supeene, C. R. Koch, and S. Bhattacharjee, "Deformation of a droplet in an electric field: Nonlinear transient response in perfect and leaky dielectric media," J. Colloid Interf. Sci., vol. 318, pp. 463-476, Feb. 2008

[15] O. Ghzaian, K. Adamiak, and G.S.P. Castle, "Spreading and retraction control of charged dielectric droplets," Colloid. Surf. A: Physicochem. Eng. Aspects, vol. 448, pp. 23-33, 2014

[16] O. Ghzaian, K. Adamiak, and G. S. P. Castle, "Numerical simulation of electrically deformed droplets less conductive than ambient fluid," Colloid. Surf. A: Physicochem. Eng. Aspects, vol. 423, pp. 27-34, Apr. 2013.

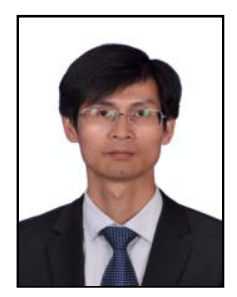

Wei-Wei Xu was born in Hubei, China, in October, 1982. He is a Ph.D. candidate in University of Science and Technology of China. He has received his bachelor degree in mechanical engineering in 2005 from Wuhan Institute of Technology, Wuhan, Hubei province, China. His education also includes the degree of Master degree in safety technology and engineering in 2011 from China University of Mining and Technology, Xuzhou, Jiangsu province, China. His previous work experience includes the position of safety engineer with SAIC Volkswagen Automotive Co., Ltd., Yangzhou, Jiangsu province, China. His main research interest is in the field of water-based paint materials fire mechanism and fire prevention, in particular the fluid dynamic. Aspect of numerical (FEA) modeling is of interest.

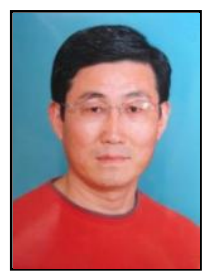

Yong-Ming Zhang received bachelor, master and $\mathrm{Ph} . \mathrm{D}$. in nuclear electronics from University of Science and Technology of China, Anhui province, China. He is a professor of the State Key Laboratory of Fire Science at University of Science and Technology of China.

His main research interest is in the field of theory and technology of fire detection, special environment fire detection, physical information detection and processing technology, and application of nuclear detection and analysis technology. He is the author of many journal publications, numerous conference papers. He won second prize in Anhui Province Science and Technology in 2011.

$\mathrm{He}$ is the member of Anhui Province Institute of electrical fire prevention branch of the Committee since 2004. 